\title{
Diagnosis and surgical repair of entero-cystocele in a cat
}

\author{
A. Rizk* and A. Samy \\ Department of Surgery, Anaesthesiology and Radiology, Faculty of Veterinary Medicine, Mansoura University, \\ Mansoura, Egypt
}

\begin{abstract}
A five-month-old male Shiraze cat was referred to the Mansoura Veterinary Teaching Hospital of Mansoura University, Mansoura, Egypt for evaluation of a 5-days history of swelling in the caudal ventral abdomen after trauma, persistent straining and inability to urinate. Clinical examination, abdominal ultrasonography and exploratory laparotomy revealed herniation and herniorraphy was attempted. The herniated organs were urinary bladder and intestine through an abdominal wall defect. Clinical signs resolved after surgical reduction of the intestine and urinary bladder. Follow-up by telephone three months postoperatively confirmed that the cat had no problems with defecation or micturition. Entero-cystocele in a young cat after trauma has not been reported previously to the author's knowledge.
\end{abstract}

Keywords: Cat, Cystocele, Enterocele, Hernia.

\section{Introduction}

Hernia is the abnormal protrusion of a part of an organ, or organs from their normal anatomical location through an abnormal defect or anatomical hole (Read and Bellenger, 2003).

Traumatic abdominal wall hernias are protrusions of abdominal contents through a trauma-induced defect in the abdominal body wall. Blunt trauma has been reported to be the most common cause of traumatic herniation in dogs and cats (Kraus, 1990; Shaw et al., 2003).

Perineal and inguinal herniation of the urinary bladder both occur in the cat (Risselada et al., 2003; Zulauf et al., 2007). Rupture of an umbilical hernia and subsequent bladder herniation have also been reported in humans (Pandey et al., 2008). Umbilical rupture with bladder herniation is not common phenomenon in the veterinary literature. Rupture of the prepubic tendon is also commonly associated with herniation of the urinary bladder from the caudal abdominal cavity in cats (Beittenmiller et al., 2009). Urinary bladder herniation through a caudoventral abdominal wall defect also has been reported in cats (Neville-Towle and Sakals, 2015).

There is little information about acquired abodominal herniation in a cat with prolapse of the intestine and urinary bladder, therefore, the case report here describes herniation of the urinary bladder and intestine through abdominal wall defect resulted from trauma in a young cat.

\section{Case Details}

A five-month-old, male, Shiraze cat weighted $3 \mathrm{~kg}$ was referred to Mansoura Veterinary Teaching Hospital of Mansoura University, Egypt with a 5- day history of trauma (car accident), The owner of the cat noticed ventral abdominal swelling at the day of trauma while the persistent straining and inability to urinate appeared since the next day of trauma. Clinical examination, abdominal ultrasonography and exploratory laparotomy were performed (Fig. 1a,b).

For exploratory coeliotomy, the cat was intramuscularly (IM) anesthetized with a mixture of xylazine HCL at a dose of $1 \mathrm{mg} / \mathrm{kg}$ (Xylaject, ADWIA, Egypt) and Ketamine HCL (Ketamine 5\%, Sigma-Tec, Egypt) at a dose of $10 \mathrm{mg} / \mathrm{kg}$. A caudal ventral midline incision was performed. After dissection of the subcauetouns abdominal fat, the intestine and urinary bladder was observed extra-abdominal in the left femoral area (Fig. 2a). A $3 \mathrm{~cm}$ length abdominal defect (hernia ring) was detected in the caudal abdominal area (Fig. 2a). The defect was just to the left of the midline but the left inguinal ring was palpably separate. Both the intestine and the urinary bladder were assessed to be viable and were reduced without complication. The external rectus abdominal muscle was closed with 3-0 PDS (Unicryl M, Unimed, Kingdom of Saudi Arabia) in a simple continuous pattern (Fig. 2b). The subcutaneous tissue and skin were closed routinely.

For the first 24 hours postoperatively, the cat was kept on intravenous fluid therapy. Antibiotic prophylaxis comprising cefotaxime sodium (Cefotax Vial, EPICO, Egypt) at a dose of $50 \mathrm{mg} / \mathrm{kg}$ IM twice a day was given for seven days. During first 48 hours postoperatively, the cat received Ketoprofen (Ketofan, Amriya, Egypt) in a dose of $2.2 \mathrm{mg} / \mathrm{kg}$ IM twice a day for pain relief.

\section{Discussion}

The cat's owner mentioned that, he paid a visit to a veterinarian who diagnosed it as just a traumatic abdominal swelling, after that the case was admitted to our Faculty Hospital approximatelythe $5^{\text {th }}$ day after

*Corresponding Author: Dr. Awad Rizk. Faculty of Veterinary Medicine, Mansoura University, Mansoura, Egypt. 


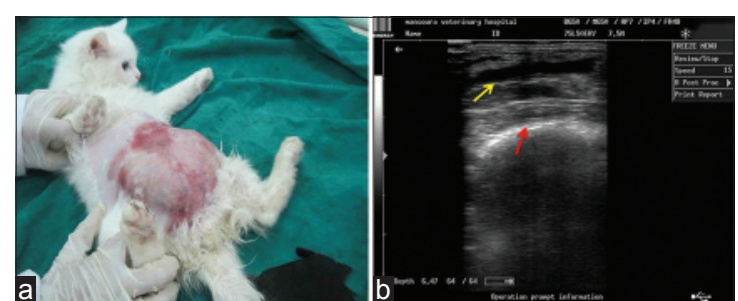

Fig. 1. (a) Five-month-old male Schraze cat with a large soft ventral swelling at the caudal abdomen. (b) Sonogram of abdominal mass in a cat showed a herniated urinary bladder (yellow arrow), omentum and the abdominal muscles (red arrow).

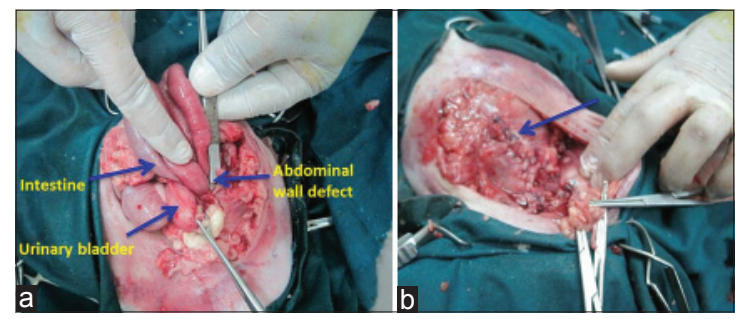

Fig. 2. Intraoperative view after dissection of the subcauetouns abdominal fat. (a) Abdominal wall defect, herniated intestinal loops, and urinary bladder (blue arrows). (b) Closure of the rectus abdominal muscle with 3-0 PDS in a simple continuous pattern (blue arrow).

trauma with persistent straining and inability to urinate. Clinical examination, abdominal ultrasonography and exploratory laparotomy were performed.

At physical examination, the cat was mildly depressed, he had a large soft swelling in the caudal ventral abdominal region (Fig. 1a).

Abdominal ultrasonographic examination revealed ventral extra-abdominal herniation of the urinary bladder and intestine. The bladder was not visible in the abdominal cavity. We found a fluctuant mass (fluidfilled cavity) in the extra-abdominal area under the skin of the ventral caudal abdomen contained anechoic fluid. The typical urinary bladder wall structure (hyperreflective, hypoechoic and hyperechoic lines) was identified (Fig. 1b). An aspiration of the extraabdominal fluid-filled structure revealed a light yellow, serous fluid. On suspicion that this structure contained urine.

The post-operative outcome revealed that, the cat was bright and alert; it drank and urinated spontaneously during the first day postsurgery. It passed normal stool on the third day. Follow-up by telephone three months postoperatively confirmed that the cat had no problems with defecation or micturition.

This report describes herniation of both intestine and the urinary bladder through an abdominal wall defect. Whilst reports of urinary bladder herniation through various abdominal wall defects have previously been described in the cat (Shaw et al., 2003 and NevilleTowle and Sakals, 2015). Our case was different from the hernia reported by Neville-Towle and Sakals (2015), as the contents of hernia in our case were urinary bladder and omentum, additionally it appeared after history of trauma, hence, it was acquired. Based on authors knowledge, the location of this defect and hernial contents of our case with known history of trauma, has not been described earlier.

In this case, the hernia was caused by a car blow to the abdomen. The blow was strong enough to tear a defect in the abdominal muscles. The small intestine and urinary bladder went through this defect and were trapped between the muscle and the underside of the skin. This needs to be corrected because the blood supply to the intestines can be compromised while entrapped in this abnormal location. This will cause a segment of the intestines to die with a possiblility of subsequent loss of life. Herniation of the urinary bladder has been described in one cat having a traumatic abdominal wall hernia (Hauptman and Hurd, 1978) and in another cat having a retroflection of the bladder within a perineal hernia (Risselada et al., 2003).

We concluded that the acquired herniation of the urinary bladder through abdominal wall defect has not been reported previously in cats to the author's knowledge, therefore, it should be considered in the differential diagnosis of caudal ventral abdominal swellings in cats following trauma.

\section{References}

Beittenmiller, M.R., Mann, F.A., Constantinescu, G.M. and Luther, J.K. 2009. Clinical anatomy and surgical repair of prepubic hernia in dogs and cats. J. Am. Anim. Hosp. Assoc. 45, 284-290.

Hauptman, J. and Hurd, B. 1978. Herniation of the urinary bladder and diaphragmatic hernia in a cat. J. Am. Vet. Med. Assoc. 172, 164-165.

Kraus, K.H. 1990. Traumatic abdominal hernias. In: Bojrab, M., Birchard, S., Tomlinson, J., eds. Current techniques in small animal surgery. $3^{\text {rd }}$ ed. Philadelphia: Lea and Febiger, pp: 499-453.

Neville-Towle, J. and Sakals, S. 2015. Urinary bladder herniation through a caudoventral abdominal wall defect in a mature cat. Can. Vet. J. 56, 934-936.

Pandey, A., Kumar, V., Gangopadhyay, A.N. and Upadhyaya, V.D. 2008. Eviscerated urinary bladder via ruptured umbilical hernia: A rare occurrence. Hernia. 12, 317-319.

Read, R.A. and Bellenger, C.R. 2003. Hernias. In: Textbook of Small Animal Surgery. $3^{\text {rd }}$ ed. Ed. D. SLATTER. Elsevier Science, Philadelphia, pp: 446-448.

Risselada, M., Kramer, M., Van de Velde, B., Polis, I. 
and Gortz, K. 2003. Retroflexion of the urinary bladder associated with a perineal hernia in a female cat. J. Small Anim. Pract. 44, 508-510.

Shaw, S.R., Rozanski, E.A. and Rush, J.E. 2003. Traumatic body wall herniation in 36 dogs and cats.
J. Am. Anim. Hosp. Assoc. 39, 35-46.

Zulauf, D., Voss, K. and Reichler, I.M. 2007. Herniation of the urinary bladder through a congenitally enlarged inguinal canal in a cat. Schweiz Arch. Tierheilk. 149, 559-562. 